Feldhoff, Tobias; Reh, Sabine; Klieme, Eckhard; Mattes, Monika; Wurster, Sebastian; Steinert, Brigitte; Dohrmann, Julia; Schmid, Christine

\title{
Schulkulturen im Wandel - Potentiale und erste Erkenntnisse zur Untersuchung von Schulkulturen im Wandel
}

van Ackeren, Isabell [Hrsg.]; Bremer, Helmut [Hrsg.]; Kessl, Fabian [Hrsg.]; Koller, Hans Christoph [Hrsg.]; Pfaff, Nicolle [Hrsg.]; Rotter, Caroline [Hrsg.]; Klein, Dominique [Hrsg.]; Salaschek, Ulrich [Hrsg.]: Bewegungen. Beiträge zum 26. Kongress der Deutschen Gesellschaft für Erziehungswissenschaft. Opladen; Berlin; Toronto : Verlag Barbara Budrich 2020, S. 433-445. - (Schriften der Deutschen Gesellschaft für Erziehungswissenschaft (DGfE))

Quellenangabe/ Reference:

Feldhoff, Tobias; Reh, Sabine; Klieme, Eckhard; Mattes, Monika; Wurster, Sebastian; Steinert, Brigitte; Dohrmann, Julia; Schmid, Christine: Schulkulturen im Wandel - Potentiale und erste Erkenntnisse zur Untersuchung von Schulkulturen im Wandel - In: van Ackeren, Isabell [Hrsg.]; Bremer, Helmut [Hrsg.]; Kessl, Fabian [Hrsg.]; Koller, Hans Christoph [Hrsg.]; Pfaff, Nicolle [Hrsg.]; Rotter, Caroline [Hrsg.]; Klein, Dominique [Hrsg.]; Salaschek, Ulrich [Hrsg.]: Bewegungen. Beiträge zum 26. Kongress der Deutschen Gesellschaft für Erziehungswissenschaft. Opladen; Berlin; Toronto : Verlag Barbara Budrich 2020, S. 433-445 - URN: urn:nbn:de:0111-pedocs-192594 - DOI: 10.25656/01:19259

https://nbn-resolving.org/urn:nbn:de:0111-pedocs-192594 https://doi.org/10.25656/01:19259

in Kooperation mit / in cooperation with:

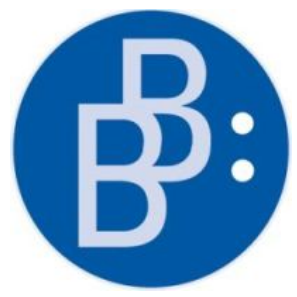

https://www.budrich.de

\section{Nutzungsbedingungen}

Dieses Dokument steht unter folgender Creative Commons-Lizenz: http://creativecommons.org/licenses/by-sa/4.0/deed.de - Sie dürfen das Werk bzw. den Inhalt vervielfältigen, verbreiten und öffentlich zugänglich machen sowie Abwandlungen und Bearbeitungen des Werkes bzw. Inhaltes anfertigen, solange sie den Namen des Autors/Rechteinhabers in der von ihm festgelegten Weise nennen und die daraufhin neu entstandenen Werke bzw. Inhalte nur unter Verwendung von Lizenzbedingungen weitergeben, die mit denen dieses Lizenzvertrags identisch, vergleichbar oder kompatibel sind.

Mit der Verwendung dieses Dokuments erkennen Sie die Nutzungsbedingungen an.

\section{Terms of use}

This document is published under following Creative Commons-License http://creativecommons.org/licenses/by-sa/4.0/deed.en - You may copy distribute and transmit, adapt or exhibit the work or its contents in public and alter, transform, or change this work as long as you attribute the work in the manner specified by the author or licensor. New resulting works or contents must be distributed pursuant to this license or an identical or comparable license.

By using this particular document, you accept the above-stated conditions of use.

\section{Kontakt / Contact:}

peDOcs

DIPF | Leibniz-Institut für Bildungsforschung und Bildungsinformation

Informationszentrum (IZ) Bildung

E-Mail: pedocs@dipf.de

Internet: www.pedocs.de

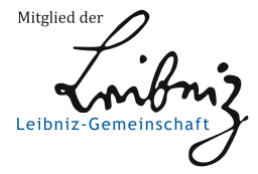




\section{Bewegungen}

Beiträge zum 26. Kongress der Deutschen Gesellschaft für Erziehungswissenschaft

Isabell van Ackeren, Helmut Bremer, Fabian Kessl, Hans Christoph Koller, Nicolle Pfaff, Caroline Rotter, Dominique Klein, Ulrich Salaschek (Hrsg.)

Schriften der Deutschen Gesellschaft für Erziehungswissenschaft

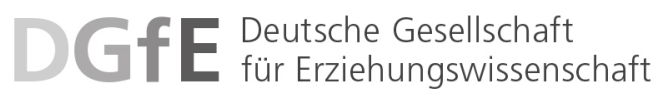


Isabell van Ackeren

Helmut Bremer

Fabian Kessl

Hans Christoph Koller

Nicolle Pfaff

Caroline Rotter

Dominique Klein

Ulrich Salaschek (Hrsg.)

\section{Bewegungen}

Beiträge zum 26. Kongress der Deutschen Gesellschaft für Erziehungswissenschaft

Verlag Barbara Budrich

Opladen • Berlin • Toronto 2020 
Bibliografische Information der Deutschen Nationalbibliothek

Die Deutsche Nationalbibliothek verzeichnet diese Publikation in der Deutschen Nationalbibliografie; detaillierte bibliografische Daten sind im Internet über

http://dnb.d-nb.de abrufbar.

(C) 2020 Dieses Werk ist bei der Verlag Barbara Budrich GmbH erschienen und steht unter der Creative Commons Lizenz Attribution-ShareAlike 4.0 International (CC BY-SA 4.0):

https://creativecommons.org/licenses/by-sa/4.0/.

Diese Lizenz erlaubt die Verbreitung, Speicherung, Vervielfältigung und Bearbeitung bei

Verwendung der gleichen CC-BY-SA 4.0-Lizenz und unter Angabe der UrheberInnen, Rechte, Änderungen und verwendeten Lizenz.

www.budrich.de

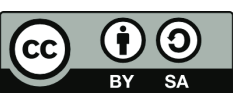

Dieses Buch steht im Open-Access-Bereich der Verlagsseite zum kostenlosen Download bereit (https://doi.org/10.3224/84742385).

Eine kostenpflichtige Druckversion (Print on Demand) kann über den Verlag bezogen werden. Die Seitenzahlen in der Druck- und Onlineversion sind identisch.

$$
\begin{array}{ll}
\text { ISBN } & 978-3-8474-2385-0 \text { (Paperback) } \\
\text { eISBN } & 978-3-8474-1553-4 \text { (PDF) } \\
\text { DOI } & 10.3224 / 84742385
\end{array}
$$

Druck: paper \& tinta, Warschau

Printed in Europe auf säurefreiem und alterungsbeständigem Papier

Umschlaggestaltung: Bettina Lehfeldt, Kleinmachnow - www.lehfeldtgraphic.de

Redaktion und Satz: Fabian Auer, Wuppertal

Typographisches Lektorat: Anja Borkam, Jena - kontakt@lektorat-borkam.de 


\section{Inhalt}

Hans-Christoph Koller

Vorwort

Fabian Kessl, Nicolle Pfaff, Isabell van Ackeren, Helmut Bremer,

Hans-Christoph Koller, Carolin Rotter, Dominique Klein, Ulrich Salaschek

Einleitung .

Käte Meyer-Drawe

Bewegungen: Viele Gemeinsamkeiten und noch mehr Unterschiede

\section{Teil I Denkbewegungen}

[Koordination: Fabian Kessl]

Christiane Thompson

„Science, not silence“. Die Öffentlichkeit der Universität an ihren Grenzen

Barbara Rendtorff, Eva Breitenbach

Frauenbewegungen, Bildung und Erziehung - Erträge und Problematiken

Britta Behm, Anne Rohstock

Loyalität. Zur verdeckten Regulierung von Denk-Bewegungen in wissenschaftlichen

Feldern. Eine Sondierung am Beispiel der Geschichte westdeutscher Bildungsforscher .... 51

Fabian Kessl

Bewegungen an den Grenzen des Disziplinären: das Beispiel von Sozialpädagogik und Sozialer Arbeit. 71

Susann Fegter, Karen Geipel, Anna Hontschik, Bettina Kleiner, Daniela Rothe, Kim-Patrick Sabla, Maxine Saborowski

Äußerungen von Sprecher*innen in einer Gruppendiskussion. Überlegungen und Analysen aus unterschiedlichen diskurs- und subjektivierungstheoretischen Perspektiven 83

Teil II Migrationsbewegungen

[Koordination: Nicolle Pfaff]

Paul Mecheril

Gibt es ein transnationales Selbstbestimmungsrecht? Bewegungsethische

Erkundungen 101 


\section{Thomas Geier}

Integration ohne Ende. Kritische Stichworte zum monothematischen Habitus der

Migrationsdebatte in Deutschland

Marcus Emmerich, Ulrike Hormel, Judith Jording, Mona Massumi

Migrationsgesellschaft im Wandel - Bildungssystem im Stillstand?

Patricia Stošić, Benjamin Rensch

„Ja, (...) wären Sie denn nicht bereit, den Lehrerberuf aufzugeben?“

Bildungsbiographische Positionierungen muslimischer Lehramtsstudentinnen im

Spannungsfeld von Pluralismusdiskurs und Diskriminierung

Arnd-Michael Nohl

Politische Erziehung. Ein blinder Fleck der Diskussion zur politischen Bildung 161

Teil III Gesellschaftliche Entwicklungen und pädagogisches Tun

[Koordination: Fabian Kessl]

Johannes Bellmann, Dirk Braun, Martina Diedrich, Katharina Maag Merki, Marcelo Parreira do Amaral, Kate Maleike

„Wer steuert die Bildung - Wer steuert die Schule?“

Ein öffentliches Podiumsgespräch zur Eröffnung des 26. Kongresses der Deutschen

Gesellschaft für Erziehungswissenschaft

Anke Wischmann, Andrea Liesner

Neu zugewanderte Jugendliche zwischen engagierter pädagogischer Hilfe,

politischen Interessen und wirtschaftlichem Kalkül

Alisha M.B. Heinemann

Learning from below - Wissen in Bewegung. $\mathrm{Zu}$ den Möglichkeiten solidarischer

Bildungsarbeit durch den 'Funds of Knowledge-Approach'

Sebastian Wachs, Wilfried Schubarth, Ludwig Bilz

Hate Speech als Schulproblem? Erziehungswissenschaftliche Perspektiven auf ein aktuelles Phänomen

Teil IV Professionalisierung in der Lehrer*innenbildung

[Koordination: Carolin Rotter]

Alexander Gröschner

Praxisbezogene Lerngelegenheiten am Beispiel lernwirksamer

Unterrichtskommunikation. „Bewegungen“ in der Aus-, Fort- und Weiterbildung

von Lehrpersonen

Julia Košinár, Anna Laros

Orientierungsrahmen im Wandel? Berufsbiographische Verläufe zwischen Studium und Berufseinstieg 
Matthias Proske, Petra Herzmann, Markus Hoffmann

Spielfilme über Lehrer/innen als Medium der kasuistischen Lehrerbildung

Kristina Geiger, Petra Strehmel

Personalentwicklung in Kindertageseinrichtungen: Maßnahmen und Strategien

von Trägern und Einrichtungen. Ergebnisse zweier empirischer Studien 283

Christina Buschle, Tina Friederich

Weiterbildung als Motor für den Erhalt von Professionalität?

Weiterbildungsmöglichkeiten für das Kita-Personal

Nikolaus Meyer, Dieter Nittel, Julia Schütz

Was haben Erzieher*innen und Professor*innen gemeinsam? Komparative

Perspektiven auf zwei stark kontrastierende pädagogische Berufsgruppen. 309

\section{Teil V Digitalisierung}

[Koordination: Isabell van Ackeren]

Manuela Pietraß

Bildung in Bewegung. Das neue Lernpotenzial digitaler Medien

Mandy Schiefner-Rohs, Sandra Hofhues, Sandra Aßmann, Taiga Brahm

Studieren im digitalen Zeitalter. Methodologische Fragen und ein empirischer Zugriff... 337

Birgit Eickelmann, Kerstin Drossel

Lehrer*innenbildung und Digitalisierung - Konzepte und Entwicklungsperspektiven .... 349

Matthias Rohs, Manuela Pietraß, Bernhard Schmidt-Hertha

Weiterbildung und Digitalisierung. Einstellungen, Herausforderungen und Potenziale ... 363

Rudolf Kammerl, Jane Müller, Claudia Lampert, Marcel Rechlitz, Katrin Potzel

Kommunikative Figurationen - ein theoretisches Konzept zur Beschreibung von

Sozialisationsprozessen und deren Wandel in mediatisierten Gesellschaften? 377

\section{Teil VI Steuerung}

[Koordination: Dominique Klein]

Michael Schemmann

„Und sie bewegt sich doch“ - Neue Steuerung und Governance in der öffentlichen

Weiterbildung.

Katharina Maag Merki

Das Educational Governance-System im Dienste der Schulentwicklung. Oder:

Wie kann Steuerung die Weiterentwicklung von Schulen unterstützen? 405 


\section{Sigrid Hartong, Annina Förschler}

Dateninfrastrukturen als zunehmend machtvolle Komponente von Educational Governance. Eine Studie zur Implementierung und Transformation staatlicher Bildungsmonitoringsysteme in Deutschland und den USA....

Tobias Feldhoff, Sabine Reh, Eckhard Klieme, Monika Mattes, Sebastian Wurster,

Brigitte Steinert, Julia Dohrmann, Christine Schmid

Schulkulturen im Wandel - Potentiale und erste Erkenntnisse zur Untersuchung von

Schulkulturen im Wandel

Felix Berth, Mariana Grgic

Wie kam die Bildung in die Krippe? Frühe Kindertagesbetreuung im Spiegel von

Wissenschaften, Recht und individuellen Einstellungen in Westdeutschland seit den 1960er-Jahren

Teil VII Körper - Leib - Bewegung

[Koordination: Fabian Kessl \& Ulrich Salaschek]

André Gogoll, Erin Gerlach

Bewegung, Sport und Lernen - zwischen pädagogischem Wunsch und empirischer

Wirklichkeit.

Maike Groen, Hannah Jäkel, Angela Tillmann, Ivo Züchner

E-Sport - Ambivalenzen und Herausforderungen eines globalen, jugendkulturellen

Phänomens.

Nino Ferrin, Benjamin Klages

Zur Kultivierung utopischer Bewegungen. Markierungen des Nicht-Verfügbaren in der Academia.

Juliane Noack Napoles

Identität als Stillstand. Ein metaphernanalytischer Blick auf eine Nicht-Bewegung.....

\section{Teil VIII Diversity / Inklusion}

[Koordination: Nicolle Pfaff]

Barbara Asbrand, Julia Gasterstädt, Anja Hackbarth, Matthias Martens

Was bewegt Inklusion? Theoretische und empirische Analysen zu

Spannungsverhältnissen einer inklusiven Schule

\section{Nina Thieme}

Zur Charakteristik der Gesellschaft, an der im Zuge von Inklusion Teilhabe ermöglicht werden soll. Vergewisserungen und Reflexionen zu möglichen Implikationen 
Bernhard Rauh, Yvonne Brandl, Michael Wininger, David Zimmermann

Inklusionspädagogik - eine halbierte Bewegung? Psychoanalytische Perspektiven

auf ein erziehungs-wissenschaftliches Paradigma

Christian Stöger

„Aber Österreich darf nicht zurückbleiben!“ Zur Wiener Hilfsschulentwicklung um 1900 . 555

Anke Karber, Gülsen Sevdiren, Kerstin Heberle, Anne Schröter, Janieta Bartz,

Tatiana Zimenkova

Hochschuldidaktische Betrachtungen differenzreflexiver Lehrer*innenbildung.

Tanja Sturm, Benjamin Wagener, Monika Wagner-Willi

Inklusion und Exklusion im Fachunterricht. Ambivalente Relationen in Schulformen

der Sekundarstufe 1

Teil IX Soziale - pädagogische Bewegungen

[Koordination: Helmut Bremer \& Jana Trumann]

Patrick Bühler

Böse Mütter im Summer of Love. Antipädagogik und Psychotherapie in den

Siebziger-Jahren

Marcel Eulenbach, Thorsten Fuchs, Yagmur Mengilli, Andreas Walther,

Christine Wiezorek

„Ich möchte Teil einer Jugendbewegung sein“"? - Jugendkultur, Protest, Partizipation.... 613

Sabrina Schenk, Britta Hoffarth, Ralf Mayer

Populismus, Protest - und politische Bildung. Soziale Bewegung(en) in

Spannungsfeldern von Affektivität, Rationalität und Praktiken der Kritik

im öffentlichen Raum

Aziz Choudry

Activist learning and knowledge production.

Autorinnen und Autoren 653 


\section{Schulkulturen im Wandel - Potentiale und erste Erkenntnisse zur Untersuchung von Schulkulturen im Wandel}

\section{$1 \quad$ Einleitung}

Die Schule ist die Institution, die sich in besonderer Weise der Sozialisation und systematischen Qualifizierung nachwachsender Generationen für ein Leben in der Gesellschaft widmet. Diese Aufgabe erfüllt die Schule unter unterschiedlichen gesellschaftlichen Bedingungen. Um die Bedeutung solcher Konstellationen für die Schule zu verstehen, lassen sich Schulen mit ihren Rahmenbedingungen entweder komparativ (Vergleich zwischen Regionen, Systemen oder Kulturen) oder diachron (Vergleich derselben Schulen über die Zeit) analysieren. Schulen verändern sich aus unterschiedlichen Gründen und unterliegen gleichzeitig Beharrungstendenzen. Schulische Grundstrukturen sind im Sinne einer grammar of schooling (Tyack/Tobin 1993) einerseits stabil. Andererseits waren mit den gesellschaftlichen Veränderungen in Deutschland in den letzten 40 Jahren neue Anforderungen an Schulen verbunden. Dazu gehören neben Entscheidungen der Bildungspolitik auch gesellschaftliche Modernisierungsprozesse, wie die Liberalisierung und Demokratisierung der Gesellschaft, die deutsche Wiedervereinigung und die zunehmende ökonomische Globalisierung. Außerdem veränderte sich die Schülerschaft. Mit der Bildungsexpansion besuchten mehr Kinder und Jugendliche das Gymnasium oder eine Schulform, die ihnen die Erlangung der Hochschulreife ermöglicht (Autorengruppe Bildungsbericht, 2018). Im wissenschaftlichen Diskurs und in der Öffentlichkeit existieren viele Vermutungen zum Wandel von Schule und Unterricht, aber kaum empirisch abgesichertes Wissen über den Wandel von Schulkulturen über einen längeren Zeitraum.

Bildungsexpansion und Wertewandel nach 1968 lassen einen fundamentalen Wandel von Schule vermuten - aber stimmt das auch für die Ebene von Schulkultur und Unterricht? Hier scheint sich viel weniger zu verändern, als man vermuten könnte: Haben sich Schul- und Unterrichtskulturen in den letzten Jahrzehnten gewandelt? Inwiefern unterliegen die Zusammenhänge zwischen Merkmalen von Schule und Unterricht und den Lernergebnissen von 
Schüler*innen zeithistorischen Veränderungen? Wie verändern sich die Kulturen von Schulen und Unterricht und deren Zusammenhänge mit den Lernergebnissen über einen längeren Zeitraum von ca. 40 Jahren? Auf diese Fragen gibt es nicht nur im deutschsprachigen Raum kaum empirisch gestützte Antworten. Um theoretisch und empirisch fundierte Beiträge zu Kontinuität und Wandel von Schule und Unterricht zu leisten, sind zunächst zeittypische Schul- und Unterrichtskulturen zu identifizieren und die Bedingungen, Prozesse und Wirkungen im Zeitvergleich zu analysieren.

Dieser Beitrag möchte anhand von ersten Erkenntnissen aus drei Teilstudien das Potential einer Mixed-Method-Studie aus quantitativer und bildungshistorischer Perspektive zur Untersuchung von Schulkulturen im Wandel aufzeigen. Hierfür wird die Drei-Länder-Studie (DLS) von Helmut Fend als Ausgangsbasis für einen Zeitvergleich skizziert. Anschließend wird das zugrundeliegende Verständnis von Schulkultur erläutert, bevor der Stand der Forschung zum Wandel von Schulkulturen aus den Erziehungswissenschaften und ihren Nachbardisziplinen umrissen wird. Dem folgt die Darstellung erster Erkenntnisse aus Re-Analysen der DLS aus den Jahren 1978 bzw. 1979 sowie aus einer Vorstudie zum erneuten Einsatz der Erhebungsinstrumente der DLS und einer historischen Fallstudie zur Kreisstadt Wetzlar. Der Beitrag schließt mit einem Ausblick auf die geplante Mixed-Method-Studie.

\section{Die Drei-Länder-Studie von Helmut Fend als Ausgangsbasis für einen Zeitvergleich}

Eine einzigartige Möglichkeit zur Analyse des Wandels von Schule und Unterricht bietet die DLS von Helmut Fend, die Ende der 1970er Jahre in Hessen, Niedersachsen und NordrheinWestfalen an insgesamt 61 Schulen durchgeführt wurde. Fend (1982) analysierte damals im Kontext der Einführung der Gesamtschulen, inwieweit sich das traditionelle Schulsystem von den neu etablierten Gesamtschulen hinsichtlich der Qualität von Schule und Unterricht unterscheidet. Sein Befund, dass die wesentlichen Faktoren nicht durch die Schulform, sondern vielmehr durch Faktoren auf Schulebene bestimmt sind, hat zur Fokussierung auf die „Einzelschule als pädagogischer Handlungseinheit" (Fend 1988) und damit zu einem Perspektivwechsel von systembezogenen Steuerungsstrategien zu Maßnahmen der Qualitätsentwicklung von Einzelschulen in Deutschland geführt. In Fends (1982) Studien ging es nicht nur um Strukturfragen. Erstmals wurden in Deutschland die Qualität von Schule und Unterricht systematisch mit fachlichen und erzieherischen Lernergebnissen von Schüler*innen sowie sozialen und kognitiven Lernvoraussetzungen in Beziehung gesetzt. Der Vergleich zwischen den Schulen in einem dreigliedrigen Schulsystem und den Gesamtschulen bezog sich auf fachliche Lernergebnisse der Schüler*innen (z.B. Leistung in Mathematik und Englisch), erzieherische Wirkungen (z.B. Schulfreude, Selbstwirksamkeit) sowie auf pädagogische Praktiken und Merkmale der Qualität von Schulen und Unterricht (z.B. Adaptivität, Strukturiertheit, Disziplin, Unterstützung).

Fends Studie war ein Wegbereiter der Schulqualitätsforschung in Deutschland. Das Design der Studie war damals schon auf eine systematische Verbindung der verschiedenen Perspektiven von Lehrkräften und Schüler*innen angelegt. Ebenso wurde die Mehr-EbenenStruktur der Daten berücksichtigt. Fend (1982) entwickelte auf der Basis seiner Befunde ein 
mehrebenentheoretisches Modell, in dem er den Beitrag der einzelnen Akteure auf den unterschiedlichen Ebenen für die Qualität von Schule aufzeigen konnte (Fend 1988).

Ausgehend von dem wissenschaftlichen Potenzial der DLS wurden die Daten von einer interdisziplinären Projektgruppe bestehend aus der Abteilung „Bibliothek für Bildungsgeschichtliche Forschung“ und „Bildungsqualität und Evaluation“" des DIPF sowie des Arbeitsbereichs „Schulentwicklung und -effektivität“ der Johannes Gutenberg-Universität Mainz (JGU) mit aktuellen Verfahren re-analysiert und für eine Wiederholungserhebung vorbereitet. Damit soll Kontinuität und Wandel von Schule und Unterricht sowie Bedingungen, Prozesse und Wirkungen dieses Wandels seit den 1979er Jahren mit Hilfe von historiographischen und empirischen Ansätzen untersucht werden.

Auf Grundlage eines Zeitzeugen- und Experteninterviews mit Helmut Fend wurde der zeithistorische Entstehungskontext der DLS rekonstruiert und die gesellschafts-, bildungsund wissenschaftspolitischen Faktoren herausgearbeitet, die die Entstehung und Interpretation der Studie(nergebnisse) begleitet haben (Mattes/Reh 2019). Im Jahr 2017 wurden die Fragebögen und Leistungstests der DLS an 10 Schulen in Hessen und Rheinland-Pfalz pilotiert, um zu prüfen, ob das damals verwendete Instrumentarium heute noch zuverlässig einsetzbar ist und damit die methodischen Voraussetzungen für eine Follow-up-Studie an den ehemaligen Schulen der DLS gegeben sind. Beide Datenbasen werden für die Analyse der Frage nach Veränderungen von Schule und Unterricht genutzt.

\section{$3 \quad$ Schulkultur im Wandel}

Für die Definition und Analyse von Schulkultur wird auf die Ansätze von Fend (1988) und Helsper (2000/2014) zurückgegriffen. Diese Ansätze ermöglichen sowohl eine quantitative Beschreibung und Analyse des Wandels von Schulkulturen als auch seine historiographische Rekonstruktion.

\subsection{Konzepte und Zugänge zum Wandel von Schulkultur}

Fend hat seinen Ansatz zur Schulkultur aus seinen Arbeiten zum Schulklima (Fend 1977) und Analysen der DLS (Fend 1988) entwickelt. Schulkulturen werden als die geteilte Sicht auf die Funktionalität und Organisation von Schule verstanden. Schulen rekontextualisieren die gesellschaftlichen, administrativen und curricularen Vorgaben jeweils nach ihren spezifischen Voraussetzungen. Auf dieser Basis gestalten sie die sozialen Beziehungen und entwickeln ein gemeinsames Verständnis der für sie angemessenen Normen, Werte und Praktiken. Diese Merkmale von Schulkultur werden auch als Prozessqualität von Schule verstanden, die mit den fachlichen und überfachlichen Ergebnissen der Schüler*innen in Beziehung stehen und sind ausgehend von diesen empirisch quantitativ bestimmbar.

Schulkulturen werden von Helsper (2000) als ,symbolische, sinnstrukturierte Ordnungen der einzelnen Schulen“ begriffen. Dieser Kulturbegriff greift auf kulturwissenschaftliche Ansätze zurück (Kolbe et al. 2008). Helsper (2000) unterscheidet analytisch zwischen Schulkultur im Sinne einer ,sich universalisierenden und tendenziell im globalen Maßstab durchsetzenden kulturellen Form des Unterrichtens“" und den empirisch zu untersuchenden plura- 
len Schulkulturen, die sich auf lokaler Ebene als Ergebnis des Zusammenspiels von kollektiven und individuellen Akteuren gestalten (vgl. Rabenstein/Reh 2009). Schulkulturen und ihr Wandel lassen sich nach Helsper (2000) als je spezifischer Umgang mit grundlegenden Antinomien der Institution Schule rekonstruieren und anhand der vier Dimensionen Partizipationsverhältnisse, Leistungsorientierung/Handhabung der Auslesepraktiken, pädagogische Werte/Gestaltung pädagogischer Beziehungen sowie Festlegung/Kanonisierung schulischer Wissensbestände beschreiben und zeitlich vergleichen.

Die Ansätze von Fend und Helsper unterscheiden sich in der Art und Weise, wie sie Schulkulturen empirisch identifizieren und deren Genese schultheoretisch erklären. Gemeinsam ist beiden Ansätzen, dass sie die Kultur der Einzelschule als spezifische Ausgestaltung pädagogischer Prozesse, der sozialen Beziehungen, der dabei geltenden Normen und der beobachtbaren Praktiken verstehen, die das Resultat der Rezeption, Auseinandersetzung und Aneignung äußerer Strukturvorgaben durch die schulischen Akteure sind. Dadurch, dass die Dimensionen der Schulkultur von Helsper und die Konstrukte der Schul- und Unterrichtskultur von Fend hier wechselseitig aufeinander bezogen werden, können die quantitativen Analysen zum Wandel der Schulkulturen historisch kontextuiert und die historiographischen Fallstudien anhand der Ergebnisse der quantitativen Analysen eingeordnet werden.

Weder Fend noch Helsper (vgl. kritisch Böhme/Hummrich/Kramer 2015) nehmen in ihrem jeweiligen Schulkulturansatz auf die Ebene des Unterrichts systematisch Bezug. Unterrichtskulturen beziehen sich auf die jeweilige Ausgestaltung von Unterrichtsprozessen und Praktiken und der sozialen Beziehungen im Unterricht einer Schule. Über gemeinsam geteilte Normen und Wertvorstellungen und über die in einer Schule bzw. in einem Kollegium erwünschte Gestaltung von Lehr- und Lernprozessen im Unterricht und von sozialen Beziehungen beeinflusst eine Schulkultur jeweilige Unterrichtskulturen.

\subsection{Befunde zum Wandel von Schulkultur}

Im deutschsprachigen Raum gibt es keine Studie, die dezidiert den Wandel von Schulkulturen über Zeiträume von ca. 40 Jahren untersucht. Hinweise zum Wandel von Schul- und Unterrichtskulturen in Einzelschulen finden sich in Studien aus unterschiedlichen Forschungsansätzen in den Erziehungswissenschaften und ihren Nachbardisziplinen: (a) bildungsgeschichtlich in sozial- wie kulturwissenschaftlicher Perspektive als Geschichte der Beschulung; (b) aus der qualitativ-empirischen Schulforschung als Rekonstruktion der Entwicklung von Schulkulturen; (c) aus der sozialisationshistorischen Forschung als Generationenvergleich; (d) aus sozialwissenschaftlichen Trendanalysen des Schulsystems auf Basis von Indikatoren für Kontext, Input, Prozess und Outcome; sowie (e) quantitativ-empirische Panelanalysen auf Schulebene im Rahmen der Schuleffektivitäts- und Schulentwicklungsforschung.

(a) In der internationalen bildungshistorischen Forschung bezeichnet das Konzept einer grammar of schooling (Tyack/Tobin 1993) eine relative Beständigkeit und Beharrungskraft der modernen Schule und des in ihr stattfindenden Unterrichts. Der Begriff ,Grammatik' verweist auf zugrundeliegende, den Schulbetrieb strukturierende Muster sowie auf die Schwierigkeit, deren Veränderung gezielt zu steuern. So wirke die grammar of schooling wie ein Filter gegenüber allen Reformversuchen und entscheide darüber mit, welche Veränderungsimpulse in welcher Form Erfolg haben und welche nicht: "Reformers believe that their innovations will change 
schools, but it is important to recognize that schools change reforms" (Tyack/Tobin 1993: 478). Während sich die Gliederung der Bildungssysteme und Lerninhalte im Laufe der letzten Jahrhunderte durchaus sichtbar verändert hätten, seien die Formen der Organisation und Legitimierung schulischen Wissens trotz zahlreicher Reformversuche nahezu unverändert geblieben: das Prinzip des Schulfaches und eines grundlegenden Fächerkanons, die Organisation des Unterrichts durch Gruppierungsweisen der Schüler*innen, vor allem in der Jahrgangsklasse, sowie die im Klassenzimmer dominanten Interaktionsmuster, nämlich die Steuerung durch eine Lehrkraft und eine bestimmte Form des Unterrichtsgespräches (Petrat, 1979, Caruso/Reh in Druck.). Vergleichende Untersuchungen zum Unterricht in Bildungssystemen verschiedener politischer Verfassungen bestätigen die These, dass Veränderungen bildungspolitischer Kontexte und von Mustern der Unterrichtsorganisation nur lose zusammenhängen. Für den deutschsprachigen Bereich hat Kluchert (2003) zeigen können, dass der Einfluss politischer Faktoren, von Zäsuren wie System- oder Regimewechseln, auf den Unterricht eher gering einzuschätzen ist. So sei trotz anderslautender Programmatik - im Bereich der Lehrer-Schüler-Interaktion keine nennenswerte Veränderung unterrichtlicher Muster im Übergang zur Weimarer Republik zu beobachten. Auch Forschungen über die politische Sozialisation in Schulen zeigen, dass im Übergang von Nationalsozialismus und früherer DDR kein direkter Wechsel der Unterrichtsordnung aufzuweisen war - obwohl es Änderungen von Unterrichtsinhalten gab (Kluchert/Leschinsky 1998).

(b) Ausgehend von den vier Dimensionen der Schulkultur stellt Helsper (2000) Vermutungen zu Entwicklungstendenzen in den letzten Jahrzehnten an, die sich teilweise mit historischen Studien zum generellen Wandel von autoritären zu liberalen Erziehungsvorstellungen decken (vgl. Gestrich 1999, von Trotha 2008). Helsper beschreibt eine Tendenz zunehmender Partizipation der Beteiligten, eine zunehmende Universalisierung individualisierter Leistungserbringung gegenüber erzieherisch-fürsorglichen Haltungen, eine Informalisierung pädagogischer Beziehungen und eine Ausweitung von Angeboten und Entscheidungsmöglichkeiten über schulische Lehrinhalte. Allerdings sind seine empirischen Belege eher dünn; immer noch fehlen - so Helsper selbst - die entsprechenden Langzeitstudien (Helsper 2008). Vereinzelt werden bestimmte Aspekte - etwa sich ändernde Autoritätsverhältnisse in den Schulen der 1950er und 1960er Jahre - in einzelnen Studien aufgegriffen (vgl. Gass-Bolm 2005).

(c) Fend und Berger (2016) haben in einem sozialisationshistorisch ausgerichteten Ansatz die Kinder ehemaliger Teilnehmer*innen von Fends Konstanzer Jugendstudie mit einer Auswahl der Fragen zur Schule und zur Erfahrung mit Schule untersucht, die bereits in den 1970er Jahren verwendet wurden. Damit haben sie eine Art von „Generationenvergleich“ vorgenommen. Die stärkere schulische Orientierung an den Individuen, an subjektiven Bedürfnissen in pädagogischen Beziehungen deuten sie in Richtung einer „Humanisierung der schulischen Binnenkultur" (Fend/Berger 2016: 883). Die Validität dieser Befunde und des von Fend und Berger angenommenen Wandels der Schulkultur über mehrere Jahrzehnte steht und fällt mit der Aussagekraft eines solchen Generationenvergleichs. Bestätigt wird diese Interpretation durch die sozialwissenschaftliche Wertewandelforschung (vgl. z.B. Inglehardt 1989, Klages 1998, kritisch dazu allerdings zeithistorisch Dietz/Neumeier/Rödder 2014). Auch Studien aus dem Umfeld der „Intellectual History“ oder kulturgeschichtliche Untersuchungen (z.B. Maase 1992, Schildt 1995/2000, Hodenberg 2006) zum kulturellen Wandel, der sich auf allen Ebenen der Gesellschaft der Bundesrepublik bis in die 1980er Jahre hinein vollzieht, unterstützen diese Position.

(d) Im Hinblick auf Merkmale des schulischen Lernumfelds und des Schulklimas zeigen sich in Deutschland zwischen 2000 und 2009 in Trendanalysen bedeutsame Verbesserungen in der Schüler-Lehrer-Beziehung und in der Klassenführung (Hertel et al. 2010). Auf der Ebene des Fachunterrichts wurden ebenfalls positive Veränderungen in der Unterrichtsführung ermittelt, so etwa für das Fach Mathematik (Sälzer et al. 2013). Während die frühen Schulleitungsstudien 
(z.B. FIMS/TIMSS 1995, PISA 2000) Anhaltspunkte für einen eher mittelmäßigen Leistungsstand deutscher Sekundarschüler*innen erbrachten (Radisch 2008), lassen neuere PISA-Erhebungen einen positiven Trend (Klieme et al. 2010, Reiss et al. 2016) erkennen. Generell gilt, dass Veränderungen des Leistungsstandes auf Systemebene, sofern sie sich zeigen, schwach ausgeprägt sind (vgl. auch Klieme 2016). Insgesamt sind die Erkenntnisse aus Trendanalysen auf der Ebene des Schulsystems nur bedingt aussagefähig im Hinblick auf professionelles Handeln in Schule und Unterricht und Zeiträume von mehr als 15 Jahren. Zudem sagen Trendvergleiche etwas über den Wandel in der Gesellschaft und im Schulsystem insgesamt aus, aber nichts über den Wandel in einzelnen Schulen.

(e) Untersuchungen zur Entwicklung von Schul- und Unterrichtskulturen und pädagogischen Prozessen in der Schulqualitätsforschung setzen bei der Einzelschule als pädagogischer Handlungseinheit an. Studien, die über mehrere Jahre oder gar Jahrzehnte laufen (Schullängsschnitt/panel), sind in der deutschen, aber auch in der internationalen Forschung sehr selten (Klieme/ Steinert 2008). Eine Ausnahme bildete das PISA-Schulpanel 2000-2009 mit 59 Gymnasien (Bischof et al. 2013). Darin zeigte sich eine Verbesserung der Beziehungsqualität zwischen Schülern*innen und Lehrkräften, aber keine Veränderung des Leistungsniveaus; besonders interessant war, dass Maßnahmen an Einzelschulen (z.B. Einführung des Ganztags) diesen Zusammenhang positiv moderierten (Bischof et al. 2013). Längsschnittliche Untersuchungen, die Schul- und Unterrichtskulturen in den Blick nehmen, die einen Zeitraum von mehr als 10 Jahren in den Blick nehmen, liegen weder national noch international vor (Goy et al. 2010).

Insgesamt zeigt sich, dass bisher keine Studie den Wandel von Schul- und Unterrichtskulturen systematisch über einen Zeitraum von ca. 40 Jahren untersucht hat. Die skizzierten Befunde geben Hinwiese zu einzelnen Aspekten des Wandels, die sich zu drei Thesen verdichten lassen: 1. Es haben sich in den Schulen wirkende pädagogische Normen und Orientierungen verändert, was sich in einem Wandel der Vorstellungen über Leistung, Selektion und Disziplinierung erkennen lässt. 2. Die pädagogischen Beziehungen in Schulen haben sich verändert, insofern als ihr informeller Charakter und partizipative Strukturen zugenommen haben. 3. Der Kern des „Schulehaltens“ und Unterrichtens hat sich nicht grundlegend verändert. Er zeichnet sich durch beständige Unterrichtspraktiken und stabile Zusammenhänge von Prozessmerkmalen auf Schul- und Unterrichtsebene sowie deren Zusammenhänge mit schulischen Ergebnissen (z.B. Lernergebnisse und -Lernmotivation) aus. Die diesen Beziehungsmustern (im Sinne von Korrelationen) liegen Gesetzmäßigkeiten zugrunde, die langfristig recht stabil sind.

\section{$4 \quad$ Erste Erkenntnisse zur Schulkultur im Wandel}

Inzwischen wurden die Daten der DLS (3493 Schüler*innen der 9. Jahrgangsstufe und 1091 Lehrkräfte aus 61 Schulen) re-analysiert, es wurde eine Pilotierung der Fragebögen im Jahr 2017 und schließlich eine historische Fallstudie im Kreis Wetzlar, der in den 1970er Jahren eine besondere schulpolitische Situation bot, durchgeführt. 


\subsection{Erste Erkenntnisse auf Basis von Re-Analysen der DLS}

In einem ersten Schritt wurden die vorliegenden Daten hinsichtlich zentraler Aspekte der Schul- und Unterrichtskultur mit aktuellen Analyseverfahren re-analysiert. Basierend auf aktuellen Methoden wurden beispielsweise die Überzeugungen und die Kooperation von Lehrkräften im Kollegium sowie die Bedeutung der verschiedenen Ebenen im Schulsystem für die Schule und Unterricht sowie für die fachlichen (Englisch und Mathematik) und überfachlichen Ergebnisse der Schüler*innen untersucht. Die empirischen Ergebnisse wurden mit den Befunden heutiger Studien verglichen, um erste Hinweise auf Stabilität und Wandel von Schule und Unterricht herauszuarbeiten.

Ausgehend von Fends (1988) Konzept der „Einzelschule als pädagogischer Handlungseinheit" wurde die Bedeutung der Schulebene für Merkmale von Schule und Unterricht im Vergleich zu den anderen Ebenen (Schulsystem, Klasse/Kurs, Individuum) innerhalb des Mehrebensystems des Schulwesens untersucht (Wurster/Feldhoff 2019). Nach diesen Befunden gibt es die eine zentrale pädagogische Handlungseinheit nicht. Je nach Merkmal sind unterschiedliche Ebenen bedeutsam. Schul- und Unterrichtskultur entsteht durch die Interaktion der verschiedenen Merkmale und über die verschiedenen Ebenen hinweg. Im Vergleich mit Befunden aus jüngeren Schulleistungsstudien (z.B. PISA 2009) weisen Merkmale der Unterrichtskultur und die Fachleistungen in den 1970er Jahren eine höhere Variabilität auf Klassenebene auf als heute.

Die Schule als pädagogische Gestaltungseinheit setzt Spielräume in der Gestaltung von Lehr-Lernprozessen durch Lehrkräfte auf Schul- und Unterrichtsebene voraus. Ein zentraler Ansatzpunkt ist die Kooperation von Lehrkräften. Sie ermöglicht es, Anforderungen und Strategien im Unterricht über Jahrgangsstufen und Klassen hinweg abzustimmen. So könnten der Unterricht und die fachlichen und erzieherischen Lernergebnisse der Schüler*innen verbessert werden. In Re-Analysen wurde der Zusammenhang zwischen Lehrerkooperation und zentralen Merkmalen der Unterrichtskultur mit den Leistungen und dem, was in den 1970er Jahren für die Beurteilung von Schule immer wichtiger wurde (vgl. Mattes 2017) und als „Wohlfühlen“ der Schüler*innen beschrieben wurde, für die Fächer Englisch und Mathematik untersucht (Steinert et al. 2019). Die Ergebnisse zeigen, dass Lehrerkooperation je nach Fach mit einzelnen Merkmalen der Unterrichtskultur zusammenhängt. Eine höhere Kooperation im Kollegium geht im Englischunterricht mit einer stärker ausgeprägten Unterstützung der Schüler*innen, im Mathematikunterricht mit höheren Anforderungen und mehr Strukturiertheit einher. Das Wohlfühlen der Schüler*innen ist in beiden Fächern auf die genannten drei Merkmale der Unterrichtskultur zurückzuführen. Ein Zusammenhang zwischen fachlichen Leistungen und Unterrichtskultur findet sich nur für das Merkmal Anforderungen im Mathematikunterricht. Diese Befunde werfen u.a. die Frage auf, ob diese fachspezifischen Zusammenhänge zeitlich stabil sind oder auf einem Wandel durch Professionalisierung zurückzuführen sind.

Die aktuelle Forschung widmet sich insbesondere dem Einfluss fachbezogener Überzeugungen als Teil der professionellen Handlungskompetenz von Lehrkräften auf das Unterrichtshandeln und die Fachleistungen. Allgemeine pädagogische Überzeugungen, wie Fend sie in der DLS untersuchte, werden heute weniger beachtet. Deshalb wurden Re-Analysen zu Zusammenhängen zwischen allgemeinen pädagogischen Überzeugungen von Lehrkräften, 
der Adaptivität des Unterrichtshandelns und fachlichen sowie überfachlichen Lernergebnissen durchgeführt (Dohrmann et al. 2019). Pädagogische Überzeugungen von Lehrkräften hängen - vermittelt über das Unterrichtshandeln - mit den fachlichen und insbesondere überfachlichen Lernergebnissen von Schüler*innen zusammen. Ob allgemeine pädagogische Überzeugungen von Lehrkräften auch heute noch eine solche Bedeutung aufweisen, soll mittels der geplanten Neuerhebung an denselben Schulen der DLS geprüft werden.

\subsection{Erste Erkenntnisse aus einer Vorstudie zum erneuten Einsatz der Erhebungsinstrumente der DLS}

Im Jahr 2017 fand eine Pilotierung der Tests und Fragebögen an 10 Schulen in Hessen und Rheinland-Pfalz statt, um ihre methodische Aktualität zu überprüfen. Der Englischtest scheint für heutige Schüler*innen leichter zu sein, der Mathematiktest als annähernd gleich schwierig - bei einem allerdings höheren Anteil an Gymnasien in der Pilotierungsstichprobe. Analysen zur Dimensionalität, Reliabilität und Messinvarianz der Skalen zeigen eine weitgehende Stabilität der Fragebögen und Tests über die Zeit. Die Mehrzahl der Konstrukte zur Unterrichtskultur weist skalare Messinvarianz auf und ermöglicht damit den Vergleich von Mittelwerten und Zusammenhängen über die Zeit (Wurster/Feldhoff 2018). Die Mehrzahl der Konstrukte zur Schulkultur weisen metrische Messinvarianz auf und erlaubt zumindest den Vergleich von Zusammenhängen über die Zeit. Nach den Ergebnissen der Messinvarianzanalysen haben die einzelnen Items eines Konstrukts heute noch die gleiche Bedeutung wie Ende der 1970er Jahre. Die unterschiedliche Bewertung einzelner Items und damit fehlende skalare Messinvarianz bei einigen Konstrukten zur Schulkultur können in Bezug auf unterschiedliche Bewertungen einzelner Items wichtige Hinweise nicht nur für einen Wandel von Schulkulturen, sondern möglicherweise auch für grundsätzlich andere Gegenwartsdiagnosen geben, die es aus historischer Perspektive zu analysieren gilt. So etwa muss die 2017 auffällig geringere Zustimmung zu einem Item, indem es darum geht, dass ,,bislang gewohnte Schulformen und Unterrichtsmethoden“ den „Anforderungen einer modernen Welt" nicht mehr entsprechen, nicht zwangsläufig auf eine geringere Reformbereitschaft der Lehrkräfte heutzutage hinweisen. Sie könnte ihren Grund auch darin finden, dass Lehrkräfte heute der Auffassung sind, dass Schulformen und Unterrichtsmethoden sich inzwischen im positiven Sinne gewandelt haben. Zudem erfährt das Item möglicherweise keine so große Zustimmung mehr, da die Formulierung „Anforderungen der modernen Welt“ eher veraltet anmuten könnte; gegenwärtige Herausforderungen werden nicht unbedingt positiv als die einer ,modernen" Welt beschrieben. Zeichen für einen schulkulturellen Wandel finden sich dort, wo es in den Items um die Förderung schwacher Schüler geht. Entgegen der Annahme, dass gegenwärtig der Wert individueller Förderung mehr geschätzt wird als früher, zeigt sich eher eine Art Härte gegenüber schwächeren Schüler*innen, eine stärkere Orientierung an individueller Leistung, möglicherweise auch Enttäuschung über die Fördermöglichkeiten seitens der Lehrkräfte. In der DLS hat sicherlich noch die Kritik an der „Leistungsschule“, wie sie um 1968 herum ihren Ausdruck fand, nachgewirkt.

Erste Hinweise auf Kontinuität und Wandel von Unterrichtskulturen über einen Zeitraum von 40 Jahren konnten aus dem Vergleich der Profile zentraler Unterrichtsmerkmale auf Basis der DLS und der Vorstudie gewonnen werden (Steinert et al. 2018). Dabei wurde der Englischunterricht in den 9. Klassen/Kursen anhand von inhaltlichen, sozialen und sozio- 
emotionalen Merkmalen der Unterrichtskultur - Strukturiertheit, Zeitnutzung, Unterstützung - mittels einer LCA typisiert. Die ermittelten drei Unterrichtsprofile aus beiden Stichproben wurden einem Zeitvergleich unterzogen und mit den Fachleistungen und dem Wohlfühlen im Unterricht in Beziehung gesetzt. Die Profile wiesen folgende Gemeinsamkeiten auf: in beiden Stichproben gab es ein Profil mit einer überdurchschnittlichen, durchschnittlichen und unterdurchschnittlichen Ausprägung der Unterrichtskultur. Es gab keinen Zusammenhang zwischen den Unterrichtsprofilen und der Englischleistung, aber einen Zusammenhang mit dem Wohlfühlen. In dieser Hinsicht scheinen die Unterrichtskulturen über die Zeit stabil zu sein. Unterschiede gab es folgende: Die Unterrichtskulturen waren im unterdurchschnittlichen Profil in der DLS niedriger ausgeprägt als in der Vorstudie. Die Einschätzung des Wohlfühlens war in der DLS heterogener als in der Vorstudie. Dies könnte auf Verbesserungen in der sozialen und sozio-emotionalen Organisation des Unterrichts und des Wohlfühlens zurückzuführen sein.

\subsection{Erste Erkenntnisse aus einer historischen Fallstudie zum Kreis Wetzlar}

Das Beispiel Wetzlar erwies sich für Fragen nach dem Wandel der Schulkulturen als besonders fruchtbares Untersuchungsfeld. Mit dem Wetzlarer Flächenversuch waren in den 1970er Jahren die Haupt-, Realschulen und Gymnasien des gesamten Landkreises in integrierte Gesamtschulen umgewandelt worden. Die Fallstudie rekonstruiert diesen Prozess auf breiter Quellengrundlage aus Archivquellen, der regionalen und lokalen Presse, hessischen Verbandszeitschriften sowie von Zeitzeugen- bzw. Experteninterviews (Mattes 2018). Das lokalhistorische Setting erlaubt es, genau zu ermitteln, wie eine staatliche Reformintervention „,von oben“ von unterschiedlichen Akteuren vor Ort (Schulpolitiker*innen, Eltern, Schulleitungen, Lehrkräften) wahrgenommen, ausgehandelt, und umgesetzt wurde. Trotz gleicher politischer Rahmenbedingungen bestanden von Anfang an relativ große Unterschiede zwischen den einzelnen Schulen des Landkreises. Eine Gemeinsamkeit, die die meisten Schulen teilten, war eine stark ausgeprägte Leistungsdifferenzierung, in A-, B-, und C-Kurse und teilweise noch weiteren Kursstufen, die sich am dreigliedrigen Schulsystem orientierten und dieses faktisch fortführten.

Neben der Entwicklung der Wetzlarer Schulregion wurden am Beispiel zweier Schulen einzelschulische Strategien, Handlungsspielräume und Praktiken untersucht, insbesondere in Bezug auf das Ziel von Gesamtschulen, Schüler*innen unabhängig von ihrer sozialen Herkunft zu fördern. Es bildeten sich zwei Entwicklungsvarianten des Wetzlarer Schulversuchs heraus: einerseits die Variante des ,heimliches Gymnasiums“, wo eine rigide äußere Leistungsdifferenzierung praktiziert wurde und Eltern wie Lehrkräfte darauf achteten, dass das Leistungsniveau der A-Kurse in Mathematik und Englisch dem vermeintlichen Gymnasialniveau entsprach. Andererseits zeigt das zweite Beispiel, dass es für das Differenzierungsangebot durchaus schulische Handlungsspielräume gab, die mit den in Schulleitung und Kollegium vorherrschenden pädagogischen Orientierungen zusammenhingen. In dieser in einem ehemaligen Arbeiterbezirk und sozialen Brennpunkt gelegenen Schule wurde die Differenzierung nur auf zwei Leistungsniveaus - Grund- und Erweiterungskurse - praktiziert. Der Unterricht war stärker projektorientiert und die Förderung individualisierter, d.h. auf die Förderung des großen Anteils von Schüler*innen mit Migrationshintergrund ausgerichtet. Das 
hohe pädagogische Engagement machte die Schule auch für Schüler*innen aus deutschsprachigen Mittelschichtsfamilien attraktiv, die die Schule zuvor gemieden hatten. Gerade die mikrohistorischen Explorationen erwiesen sich als aufschlussreich für die Frage, wie das Zusammenwirken unterschiedlicher Faktoren den Wandel von Schule und Schulkultur fördern oder bremsen kann wie etwa die Sozialstruktur eines Einzugsgebiets und die daran gebundenen Erziehungs- und Leistungsvorstellungen, die in den Schulen vorherrschenden pädagogischen Ideen, Werte und Überzeugungen, die die inneren Prozesse und Praktiken beeinflussen ebenso wie die Generationszugehörigkeit der Lehrkräfte.

\section{$5 \quad$ Fazit und Ausblick zur geplanten Studie zum Wandel von Schulkultur}

Die DLS ist die einzige Studie in Deutschland, die - heute als Follow-up-Studie wiederholt - die Erforschung des Wandels von Schul- und Unterrichtkulturen über einen Zeitraum von 40 Jahren ermöglicht. Die vorgestellten Erkenntnisse zum Wandel von Schulkulturen auf Basis der Re-Analysen der Daten der DLS, der Vorstudie zum erneuten Einsatz der Erhebungsinstrumente und der historiographischen Vorstudie zeigen, dass Fends DLS ein idealer Ausgangspunkt für Analysen des historischen Wandels von Schul- und Unterrichtskulturen ist. Das interdisziplinäre Projekt von JGU und DIPF verfolgt das Ziel, substantielle Beiträge zu Kontinuität und Wandel der Schul- und Unterrichtskulturen vor dem Hintergrund des gesellschaftlichen Wandels seit den 1970er Jahren aus der bildungshistorischen und quantitativen Schulforschung zu leisten. Ein daraus gewonnenes Wissen über Stabilität und Wandel von einzelschulischen Kulturen stellt einen Beitrag zur Schultheorie, zum Verständnis des Funktionierens der zentralen gesellschaftlichen Bildungsinstitution dar und kann im Rahmen bildungshistorischer Forschung als Beitrag zur Identifizierung einer zeittypischen Schulkultur gelten. In historischer Perspektive werden der Wandel schulpolitischer, schulischer und pädagogischer Themen öffentlicher und professioneller Diskurse der vergangenen 40 Jahre rekonstruiert sowie der Wandel einzelner Schulen, deren Umgang mit veränderten inneren und äußeren Bedingungen in historiographischen Fallstudien anhand von Schulunterlagen, von Akten der kommunalen, regionalen und länderspezifischen Schulverwaltung, der Lokalpresse und von Zeitzeugeninterviews nachgezeichnet. Diese Rekonstruktionen dienen gleichzeitig als Interpretationsfolie für die quantitativen Analysen. In diesen sollen einerseits Stabilität und Wandel der soziokulturellen Zusammensetzung der Schülerschaft, der Schul- und Unterrichtkultur und den schulischen Ergebnissen untersucht werden. Zudem steht die Analyse der Stabilität und des Wandels der Zusammenhänge zwischen Merkmalen der Schulund Unterrichtskultur und schulischen Ergebnissen im Mittepunkt der Studie. Die Analysen der historischen und quantitativen Perspektive werden schließlich zueinander in Beziehung gesetzt und im Sinne eines Mixed-Method-Ansatzes integriert. 


\section{Literatur}

Autorengruppe Bildungsberichterstattung (2018): Bildung in Deutschland 2018. Bielefeld: wbv media.

Bischof, Linda/Hochweber, Jan/Hartig, Johannes/Klieme, Eckhard (2013): Schulentwicklung im Verlauf eines Jahrzehnts - Erste Ergebnisse des PISA Schulpanels. In: Jude, Nina/Klieme, Eckhard (Hrsg.): PISA 2009 - Impulse für die Schul-und Unterrichtsforschung. Weinheim und Basel: Beltz Juventa, S. 172-199.

Böhme, Jeanette/Hummrich, Merle/Kramer, Rolf-Torsten (Hrsg.) (2015): Schulkultur. Theoriebildung im Diskurs. Wiesbaden: VS-Verlag.

Caruso, Marcelo (2003): Biopolitik im Klassenzimmer. Zur Ordnung der Führungspraktiken in den Bayerischen Volksschulen (1869-1918). Weinheim u.a.: Beltz.

Caruso, Marcelo/Reh, Sabine (in Druck): Unterricht. In: Caruso, Marcelo/Groppe, Carola/Horn, KlausPeter/Kluchert, Gerhard/Mietzner, Ulrike (Hrsg.): Historische Bildungsforschung. Ein Handbuch. Bad Heilbrunn: Klinkhardt.

Dietz, Bernhard/Rödder, Andreas/Neumaier, Christopher (Hrsg.) (2014): Gab es den Wertewandel? Neue Forschungen zum gesellschaftlich-kulturellen Wandel seit den 1960er Jahren. München: Oldenbourg.

Dohrmann, Julia/Feldhoff, Tobias/Steinert, Brigitte/Klieme, Eckhard (2019): Überzeugungen von Lehrkräften, Adaptivität des Unterrichts und Lernergebnisse im Fach Englisch. In: Zeitschrift für Pädagogik, 65, 1, S. 56-72.

Fend, Helmut (1982): Gesamtschule im Vergleich. Bilanz der Ergebnisse des Gesamtschulversuchs. Weinheim und Basel: Beltz.

Fend, Helmut (1988): Schulqualität. Die Wiederentdeckung der Schule als pädagogische Gestaltungsebene. In: Neue Sammlung, 28, 4, S. 537-547.

Fend, Helmut/Berger, Fred (2016): Ist die Schule humaner geworden? Sozialhistorischer Wandel der pädagogischen Kulturen in Schule und Familie in den letzten 30 Jahren im Spiegel der LifE-Studie. In: Zeitschrift für Pädagogik, 62, 6, S. 861-885.

Gass-Bolm, Torsten (2005): Das Gymnasium 1945 - 1980. Bildungsreform und gesellschaftlicher Wandel in Westdeutschland. Göttingen: Wallstein.

Gestrich, Andreas (1999): Geschichte der Familie im 19. und 20. Jahrhundert. München: Oldenbourg.

Goy, Martin/Gröhlich, Carola/Strietholt, Rolf/Stubbe, Tobias C./Bos, Winfried/Kanders, Michael (2010): Panelstudien als Antworten auf Forschungsdesiderate in der Sekundarstufe I. In: Berkemeyer, Nils/Bos, Wilfried/Holtappels, Heinz Günter/McElvany, Nele/Schulz-Zander, Renate (Hrsg.): Jahrbuch der Schulentwicklung Band 16: Daten, Beispiele und Perspektiven. Weinheim: Juventa, S. 37-70.

Helsper, Werner (2000): Wandel der Schulkultur. In: Zeitschrift für Erziehungswissenschaft, 3, 1, S. 35-60.

Helsper, Werner (2008): Schulkulturen als symbolische Sinnordnungen und ihre Bedeutung für die pädagogische Professionalität. In: Helsper, Werner/Busse, Susann/Hummrich, Merle/Kramer, Rolf-Torsten (Hrsg.): Pädagogische Professionalität in Organisationen. Wiesbaden: VS Verlag, S. 115-148.

Helsper, Werner (2014): Schülerbiographie und Schülerhabitus. Zur Transformation des Schülerhabitus im Verlauf der Schülerbiographie. In: Brachmann, Jens/Lübcke, Claudia/Schwertfeger, Anja (Hrsg.): Jugend - Perspektiven eines sozialwissenschaftlichen Forschungsfeldes. Bad Heilbrunn: Julius Klinkhardt, S. 79-89. 
Hertel, Silke/Hochweber, Jan/Steinert, Brigitte/Klieme, Eckhard (2010): Schulische Rahmenbedingungen und Lerngelegenheiten im Deutschunterricht. In: Klieme, Eckhard/Artelt, Cordula/Hartig, Johannes/Jude, Nina/Köller, Olaf/Prenzel, Manfred/ Schneider, Wolfgang/Stanat, Petra (Hrsg.): PISA 2009. Bilanz nach einem Jahrzehnt. Münster: Waxmann, S. 113-151.

Hodenberg, Christina von/Siegfried, Detlef (Hrsg.) (2006): Wo $>>1968<<$ liegt: Reform und Revolte in der Geschichte der Bundesrepublik: Göttingen: Vandenhoeck \& Ruprecht.

Inglehart, Ronald (1989): Kultureller Umbruch. Wertwandel in der westlichen Welt. Frankfurt/Main: Campus-Verlag.

Klages, Helmut (1998): Wertewandel und Moralität. In: Lüschen, Günther (Hrsg.): Das Moralische in der Soziologie. Wiesbaden: VS Verlag, S. 107-125.

Klieme, Eckhard/Steinert, Brigitte (2008): Schulentwicklung im Längsschnitt. Ein Forschungsprogramm und erste explorative Analysen. In: Prenzel, Manfred/Baumert, Jürgen (Hrsg.): Vertiefende Analysen zu PISA 2006. Wiesbaden: Springer, S. 221-238. (Zeitschrift für Erziehungswissenschaft, Sonderheft; 10).

Klieme, Eckhard (2016): Schulqualität, Schuleffektivität und Schulentwicklung: Welche Erkenntnis eröffnet empirische Forschung? In: Steffens, Ulrich/Bargel, Tino (Hrsg.): Schulqualität - Bilanz und Perspektiven. Münster: Waxmann, S. 45-64.

Klieme, Eckhard/Artelt, Cordula/Hartig, Johannes/Jude, Nina/Köller, Olaf/Prenzel, Manfred/Schneider, Wolfgang (Hrsg.) (2010): PISA 2009. Bilanz nach einem Jahrzehnt. Münster: Waxmann.

Kluchert, Gerhard/Leschinsky, Achim (1998): Schule in der Transformation - Transformation der Schule? Was man aus. Gesprächen mit ehemaligen Schülern über die Schule "zwischen zwei. Diktaturen" erfahren kann. In: Zeitschrift für Pädagogik, 44, 4, S. 543-564.

Kluchert, Gerhard (2003): Die Entwicklung der Lehrer-Schüler-Interaktion und die Bildungswachstumsschübe. Zur inneren Schulreform im 20. Jahrhundert. In: Zeitschrift für Pädagogik, 49, 1, S. 47-60.

Kolbe, Fritz/Reh, Sabine/Fritzsche, Bettina/Idel, Till-Sebastian/Rabenstein, Kerstin (2008): Lernkultur. Überlegungen zu einer kulturwissenschaftlichen Grundlegung qualitativer Unterrichtsforschung. In: Zeitschrift für Erziehungswissenschaft, 11, 1, S. 125-143.

Maase, Kaspar (1992): BRAVO Amerika. Erkundungen zur Jugendkultur der Bundesrepublik in den fünfziger Jahren. Hamburg: Junius.

Mattes, Monika (2017): Von der Leistungs- zur Wohlfühlschule? In: Reh, Sabine/Glaser, Edith/Behm, Britta/Drope, Tilman (Hrsg.): Wissen machen. Beiträge zu einer Geschichte erziehungswissenschaftlichen Wissens in Deutschland zwischen 1945 und 1990. Basel und Weinheim: Beltz Juventa, S. 187-206. (Zeitschrift für Pädagogik, Beiheft; 63).

Mattes, Monika (2018): Gesamtschule im Flächenversuch. Erfahrungen mit einem bildungspolitischen Experiment im hessischen Wetzlar 1965-1990. In: Hoffmann-Ocon, Andreas/Criblez, Lucien/Groppe, Carola (Hrsg.): Schwerpunkt. Scheinbarer Stillstand - pädagogische Diskurse und Entwicklungen in den Achtzigerjahren. Bad Heilbrunn: Klinkhardt, S. 29-58.

Mattes, Monika/Reh, Sabine (2019): Entstehung und Durchführung der Gesamtschul-Studien in den 1970er Jahren. In: Zeitschrift für Pädagogik, 65, 1, S. 9-24.

Petrat, Gerhardt (1979): Schulunterricht. Seine Sozialgeschichte in Deutschland 1750 bis 1850 . München: Ehrenwirth.

Rabenstein, Kerstin/Reh, Sabine (2009): Einzelschulforschung als rekonstruktiv-qualitative Sozialforschung. In: Mertens, Gerhard/Forst, Ursula/Böhm, Winfried/Ladenthin, Volker (Hrsg.): Handbuch der Erziehungswissenschaft, Band 2. Paderborn u.a.: Schöningh, S. 729-737. 
Radisch, Falk (2008): Von FIMS bis PIRLS und PISA - Deutschlands Abschneiden bei internationalen Schulleistungsvergleichen. In: Böttcher, Wolfgang/Bos, Wilfried/Döbert, Hans/Holtappels, Heinz Günter (Hrsg.): Bildungsmonitoring und Bildungscontrolling in nationaler und internationaler Perspektive. Münster: Waxmann, S. 183-194.

Reiss, Kristina/Sälzer, Christine/Schiepe-Tiska, Anja/Klieme, Eckhard/Köller, Olaf (Hrsg.) (2016): PISA 2015. Eine Studie zwischen Kontinuität und Innovation. Münster: Waxmann.

Schildt, Axel (1995): Moderne Zeiten. Freizeit, Massenmedien und 'Zeitgeist' in der Bundesrepublik der 50er Jahre. Hamburg: Hans Christian Verlag.

Schildt, Axel (2000): Materieller Wohlstand - pragmatische Politik - kulturelle Umbrüche. Die 60er Jahre in der Bundesrepublik. In: Schildt, Axel/Siegfried, Detlef/Lammers, Karl Christian (Hrsg.): Dynamische Zeiten. Die 60er Jahre in den beiden deutschen Gesellschaften. Hamburg: Hans Christian Verlag, S. 21-53.

Steinert, Brigitte/Dohrmann, Julia/Schmid, Christine (2019): Lehrerkooperation, Unterrichtsqualität und fachliche und überfachliche Ergebnisse von Schüler*innen in Englisch und Mathematik. Eine Re-Analyse der Drei-Länder-Studie von Helmut Fend. In: Zeitschrift für Pädagogik, 65, 1, S. 4055 .

Steinert, Brigitte/Schmid, Christine/Dohrmann, Julia (2018): Unterrichtskulturen als Profile von Unterrichtsmerkmalen im Wandel der Zeit. Vortrag gehalten auf der DGfE-Tagung am 21.03.2018 in Essen.

Steinert, Brigitte/Dohrmann, Julia/Schmid, Christine (2019): Lehrerkooperation, Unterrichtsqualität und fachliche und überfachliche Ergebnisse von Schüler*innen in Englisch und Mathematik. Eine Re-Analyse der Drei-Länder-Studie von Helmut Fend. In: Zeitschrift für Pädagogik, 65, 1, S. 4055 .

Tyack, David/Tobin, William (1993): The "Grammar" of Schooling: Why Has it Been so Hard to Change? In: American Educational Research Journal Fall, 31, 3, pp. 453-479.

Trotha, Trutz von (2008): Die bürgerliche Familie ist tot. Vom Wert der Familie und Wandel der gesellschaftlichen Normen. In: Rödder, Andreas (Hrsg.): Alte Werte - Neue Werte. Schlaglichter des Wertewandels. Göttingen: Vandenhoeck \& Ruprecht, S. 78-93.

Wurster, Sebastian/Feldhoff, Tobias (2018): Schule im Wandel: Stabilität und Veränderung von Konstrukten. Vortrag auf dem 26. Kongress der Deutschen Gesellschaft für Erziehungswissenschaft (DGfE) am 21.03.2018 in Essen.

Wurster, Sebastian/Feldhoff, Tobias (2019): Schul- und Unterrichtsqualität aus der Mehrebenenperspektive. Ist die Schule oder die Klasse die relevante pädagogische Gestaltungseinheit? In: Zeitschrift für Pädagogik, 65, 1, S. 24-39. 